\title{
Prosurvival activity for airway neutrophils in severe asthma
}

\author{
Mohib Uddin, ${ }^{1}$ Guangmin Nong, ${ }^{1}$ Jonathan Ward, ${ }^{1}$ Grégory Seumois, ${ }^{1}$ Lynne R Prince, ${ }^{2}$ \\ Susan J Wilson, ${ }^{1}$ Victoria Cornelius, ${ }^{1}$ Gordon Dent, ${ }^{1}$ Ratko Djukanović ${ }^{1}$
}

\section{See Editorial, p 665}

- Supplementary methods and a figure are published online only. To view these files please visit the journal online (http:// thorax.bmj.com).

${ }^{1}$ Southampton NIHR Respiratory Biomedical Research Unit, Southampton Wellcome Trust Clinical Research Facility, Division of Infection, Inflammation and Immunity, University of Southampton School of Medicine, Southampton General Hospital, Southampton, UK

${ }^{2}$ Academic Unit of Respiratory Medicine, School of Medicine \& Biomedical Sciences, University of Sheffield, Royal Hallamshire Hospital, Sheffield, UK

\section{Correspondence to}

Professor Ratko Djukanović, Southampton NIHR Respiratory Biomedical Research Unit,

Division of Infection,

Inflammation and Immunity, School of Medicine, University of Southampton School of Medicine, Mailpoint 810, Level F, Sir Henry Wellcome Laboratories, South Block, Southampton General Hospital, Southampton S016 6YD, UK: r.djukanovic@southampton.ac. uk

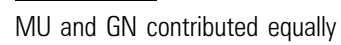
to this work.

Received 13 July 2009 Accepted 1 June 2010

\section{ABSTRACT}

Background Airway neutrophilia is a recognised feature of chronic severe asthma, but the mechanisms that underlie this phenomenon are unknown. Evidence for factors present in airway secretions that prolong neutrophil survival has been sought and it has been hypothesised that these might be augmented in neutrophilic asthma.

Methods Non-smoking subjects with severe asthma (SA) or mild asthma (MA) and healthy control subjects (HC) underwent sputum induction. The SA group was subdivided into subjects with neutrophil counts above (SA-high) and those within the normal range (SA-low). Apoptotic neutrophils were enumerated in the cellular phase while the fluid phase was assessed for its ability to prolong the in vitro survival of blood-derived neutrophils using morphometric and flow cytometric analyses.

Results There was a significant difference between all four subject groups with respect to the percentage of apoptotic sputum neutrophils (Kruskal-Wallis, $p=0.042)$. Cuzick test showed a highly significant $(p=0.008)$ trend towards decreasing numbers of apoptotic neutrophils across the four groups with increasing asthma severity and neutrophil count. The sputum antiapoptotic activity was also different between the groups $(p=0.039)$, with a highly significant $(p=0.005)$ decreasing trend across the four groups. The survival effect could not be inhibited by blocking selective chemotaxin receptors, neutralising neutrophil survival factors, inhibiting phosphatidylinositol-3-kinase (using LY294002) or with pertussis toxin pretreatment.

Similarly, it could not be explained by lipopolysaccharide contamination or by the presence of inhaled

corticosteroids in sputum.

Conclusions These data demonstrate the capacity of as yet unidentified factor(s) in the airways of subjects with asthma to delay human neutrophil apoptosis and extend their lifespan as a potential mechanism contributing to unresolving airways neutrophilia in severe asthma.

\section{INTRODUCTION}

Accumulating evidence points to an important role for neutrophils during acute exacerbations of asthma and in severe forms of the disease ${ }^{1-3}$ where raised neutrophil numbers have been observed in bronchoalveolar lavage (BAL), induced sputum and both bronchial and transbronchial biopsies. ${ }^{1} 45$ While increased concentrations of neutrophil-active mediators interleukin 8 (IL-8), ${ }^{6}{ }^{7}$ leukotriene $\mathrm{B}_{4}$ $\left(\mathrm{LTB}_{4}\right){ }^{78}$ granulocyte-macrophage colony-stimulating factor $(\mathrm{GM}-\mathrm{CSF})^{9}$ and tumour necrosis factor $\alpha(\mathrm{TNF} \alpha)^{10}$ have been detected in BAL and plasma of patients with severe asthma, a clear mechanistic association between these mediators and airways neutrophilia has not yet been demonstrated.

Theoretically, the observed neutrophilia in the airways of those with asthma could be due to increased chemotactic activity produced in the inflamed airways or longer survival due to locally produced factors, such as TNF $\alpha$ and GM-CSF, which delay their apoptosis. ${ }^{11}$ It is increasingly recognised that diverse phenotypes exist in asthma. ${ }^{12}{ }^{13}$ It is also known that there is marked variability in airway cytokine/chemokine/growth factor profiles, expression of receptors and signalling pathways, ${ }^{4}{ }^{4-17}$ all of which could represent different phenotypes. In a subpopulation of subjects with severe asthma, partial or complete steroid resistance is seen. ${ }^{2}$ Paradoxically, corticosteroid treatment itself could contribute to airway neutrophilia in poorly controlled asthma by promoting neutrophil survival. ${ }^{18-20}$ While the exact role of neutrophils in asthma is still unknown, an extended lifespan and/or failed clearance of apoptotic neutrophils could enhance the proinflammatory potential of these cells to cause lung tissue damage and to impede the resolution of asthmatic inflammation. ${ }^{21}$

In order to elucidate the mechanisms that lead to airways neutrophilia in severe asthma, we have conducted a cross-sectional study of subjects with mild asthma treated with $\beta_{2}$-agonists alone and those with severe asthma on high dose inhaled (ICS) and oral corticosteroids (OCS), using healthy individuals as control subjects. We have hypothesised that raised neutrophil numbers in more severe asthma are the result of their prolonged survival promoted by factors released by inflammatory and structural cells of the airways. In order to test this hypothesis, we first quantified the extent of in vivo apoptosis by enumerating sputum neutrophils that display characteristic morphological features of apoptosis. Further evidence of increased survival in asthmatic airways was then sought by studying whether factors present in the fluid phase of sputum, which samples the epithelial lining fluid (ELF), could alter the proportions of neutrophils that become apoptotic in neutrophil cultures. Any effect of residual corticosteroids in sputum samples was tested by pretreating neutrophils with the glucocorticoid (GC) receptor antagonist, mifepristone (RU486), added to neutrophil survival assays. ${ }^{22}$ The mechanisms underlying any proneutrophil survival effect of the sputum fluid phase were then studied using selective blockers for mediator pathways known to delay neutrophil apoptosis. 


\section{METHODS \\ Subjects}

Non-smoking volunteers, classified as severe atopic asthmatics (SA; $\mathrm{n}=15)$, mild atopic asthmatics (MA; $\mathrm{n}=11$ ) or healthy control subjects (HC $(n=12)$ using established GINA (Global Initiative for Asthma) guidelines, were enrolled. The SA subjects were further divided into two subgroups according to the percentage of neutrophils in sputum using the $65 \%$ cut-off for normal neutrophil counts reported by Belda and co-workers ${ }^{23}$ to define SA with high counts (SA-high) and SA with low counts (SA-low) (for criteria see the online supplement). The study was approved by the Southampton and South West Hampshire Ethics committee and all subjects were recruited using advertisements in the media, either directly or via a departmental database.

\section{Sputum induction and processing}

Sputum induction was conducted using guidelines of the ERS (European Respiratory Society) Task Force on induced sputum ${ }^{24}$ with $4.5 \%$ hypertonic saline as described previously. ${ }^{25}$

\section{Sputum cell counts and quantification of apoptotic neutrophils} Immunocytochemistry was applied to sputum cell phase cytospins to identify neutrophils ${ }^{26}$ using mouse monoclonal antineutrophil elastase antibody, followed by secondary rabbit antimouse bionylated immunoglobulin $\mathrm{G}(\mathrm{IgG})$, streptavidin-AP (alkaline phosphatase) and Fast Red, and counterstained with haematoxylin. Apoptotic neutrophils were defined as those positively immunostained cells that also exhibited cell shrinkage, nuclear condensation and fragmentation, plasma membrane ruffling and blebbing, features known to be characteristic of apoptotic cells and widely used for their identification. ${ }^{21}$

\section{In vitro assessment of antiapoptotic activity of the sputum fluid phase}

Neutrophils were obtained from normal healthy donors, purified to $>98 \%$ purity using standard methods known not to activate neutrophils $\mathrm{s}^{27}$ and resuspended at $4 \times 10^{6}$ cells $/ \mathrm{ml}$ in RPMI 1640 with $20 \%$ fetal calf serum (FCS) plus $2 \%$ L-glutamine, sodium pyruvate supplemented with $50 \mathrm{U} / \mathrm{ml}$ streptomycin, and penicillin $\mathrm{G}$, and incubated at $37^{\circ} \mathrm{C}$ in $5 \% \mathrm{CO}_{2}$. For all subjects, experiments were carried out in this medium in the presence or absence of sputum fluid phase (final concentration 1:20, shown in preliminary experiments to be optimal in prolonging neutrophil survival), or lipopolysaccharide (LPS) $(10 \mathrm{ng} / \mathrm{ml})$, a known antiapoptotic factor for neutrophils, as a positive control. Experiments were conducted in parallel in the presence of the GC receptor antagonist, mifepristone $(1 \mu \mathrm{M})$ in order to control for any residual corticosteroids present in sputum. In separate culture wells, the effect of mifepristone was controlled by testing its ability to abrogate the inhibitory effects of dexamethasone $(100 \mathrm{nM})$. Dexamethasone and mifepristone were dissolved in ethanol (final EtOH concentration $0.01 \%(\mathrm{v} / \mathrm{v}$ ) found not to affect constitutive neutrophil apoptosis (data not shown)). After $22 \mathrm{~h}$ culture, neutrophils were centrifuged at $300 \mathrm{~g}$ for $10 \mathrm{~min}$ at $4^{\circ} \mathrm{C}$, washed twice and resuspended with $10 \%$ heat-inactivated FCS/phosphate-buffered saline (PBS). Cytocentrifuged preparations were fixed in methanol and stained with Diff-Quik: this allowed blind counting of slides and for apoptosis to be scored morphometrically as those cells with decreased size and nuclear and cytoplasmic condensation (figure S1; online supplement). Numbers of apoptotic cells were reported as percentages of total neutrophil counts. In subsequent inhibition experiments, in which inhibitors of various mediators and signalling pathways were used to block the effect of sputum on in vitro apoptosis, the extent of apoptosis was assessed by flow cytometry using fluorescein isothiocyanate (FITC)-labelled Annexin V (BD Biosciences, Oxford, UK) and a FACSCalibur with Cell Quest software (BD Biosciences) by counting 10000 events per sample. We have previously shown a strong $\left(\mathrm{r}_{\mathrm{s}}=0.70\right)$ and highly significant $(p<0.0001)$ correlation between the extent of neutrophil apoptosis measured using standard morphological criteria and the extent quantified by flow cytometry. ${ }^{27}$ For further details of all the experimental methods see the online supplement.

\section{Statistics}

All statistical analyses were performed using Prism version 5 for Windows (GraphPad Software, San Diego, California, USA) except the Cuzick test ${ }^{28}$ which was performed using STATA, Version 11.0 (StataCorp, College Station, Texas, USA). Sputum neutrophil numbers and neutrophil survival in in vitro assays were not normally distributed. These data are therefore expressed as median, IOR and range from the indicated numbers of experiments. Differences in these indices among the four groups of patients were assessed by the Kruskal-Wallis test. Where this returned a significant difference, the Cuzick nonparametric test for trend across the four groups (HC, MA, SAlow and SA-high) was performed..$^{28}$ The effects of drugs on the antiapoptotic activity of sputum supernatants on neutrophils in vitro were assessed using the paired Wilcoxon signed-rank test. A probability of 0.05 was defined as significant.

\section{RESULTS}

\section{Subject details}

Subjects' clinical characteristics and their sputum neutrophil and eosinophil counts are shown in table 1 . Forced expiratory volume in $1 \mathrm{~s}\left(\mathrm{FEV}_{1}\right)$ was significantly lower in the SA-high and SA-low groups than in the MA or HC groups (all $\mathrm{p}<0.0001$ ). Analysis of sputum revealed that the percentage of neutrophils in the SA-high group was significantly higher than in the SAlow, MA and HC groups (all $\mathrm{p}<0.0001$ ).

\section{Neutrophil apoptosis in sputum}

The percentage of apoptotic neutrophils counted in the sputum cell phase was significantly different between the four subject groups as assessed by the Kruskal-Wallis test $(p=0.042)$. Trend

Table 1 Subject characteristics

\begin{tabular}{|c|c|c|c|c|}
\hline Variables & $\begin{array}{l}\mathrm{HC} \\
(n=12)\end{array}$ & $\begin{array}{l}\mathrm{MA} \\
(n=11)\end{array}$ & $\begin{array}{l}\text { SA-high } \\
(n=6)\end{array}$ & $\begin{array}{l}\text { SA-low } \\
(n=9)\end{array}$ \\
\hline Age (years) & $43 \pm 15.5$ & $36 \pm 10.4$ & $45 \pm 7.1$ & $45 \pm 11.5$ \\
\hline Gender (M:F) & $9: 3$ & $7: 4$ & $4: 2$ & $5: 4$ \\
\hline Duration of asthma (years) & NA & $20.8 \pm 12.3$ & $33.3 \pm 12.5$ & $35.4 \pm 16.9$ \\
\hline $\mathrm{FEV}_{1}(\%$ of predicted) & $99 \pm 7.2$ & $95 \pm 12.8$ & $62 \pm 6.8^{*}$ & $72 \pm 18.8^{*}$ \\
\hline $\begin{array}{l}\text { Sputum neutrophils (\% of total } \\
\text { inflammatory cells) }\end{array}$ & $50.6 \pm 17.2$ & $36.4 \pm 16.9 \neq$ & $81.1 \pm 9.9 \dagger$ & $38.5 \pm 15.1$ \\
\hline $\begin{array}{l}\text { Sputum eosinophils (\% of total } \\
\text { inflammatory cells) }\end{array}$ & $0.2 \pm 0.4$ & $2.0 \pm 1.8 \Phi$ & $2.6 \pm 2.6 \S$ & $13.4 \pm 17.7^{*}$ \\
\hline
\end{tabular}

Data are expressed as mean and SEM.

The SA-high and SA-low groups were defined according to neutrophil counts $>65 \%$ and $<65 \%$ of total inflammatory cell counts, respectively.

${ }^{*} \mathrm{p}<0.0001$ versus MA and $\mathrm{HC}$

$t \mathrm{p}<0.0001$ versus MA, SA-low and HC.

$\neq \mathrm{p}=0.043$ versus $\mathrm{HC}$.

$\S \mathrm{p}=0.0012$ versus $\mathrm{HC}$

$\Phi p<0.0009$ versus $\mathrm{HC}$

$\mathrm{F}$, female; $\mathrm{FEV}_{1}$, forced expiratory volume in $1 \mathrm{~s} ; \mathrm{HC}$, healthy controls; $\mathrm{M}$, male; $\mathrm{MA}$, mild asthma; NA, not applicable; SA, severe asthma. 
analysis using the Cuzick test showed a highly significant decreasing trend in the ranked score of apoptotic neutrophils across the four groups with increasing asthma severity and neutrophil counts (Cuzick test for trend, $\mathrm{p}=0.008$ ) (figure 1).

\section{Effect of sputum supernatant on neutrophil apoptosis}

All sputum samples contained significant prosurvival activity, as demonstrated by their ability to reduce the degree of constitutive neutrophil apoptosis in vitro (median of $56.90 \%$, range of 19.70-85.30\%). However, the activity was significantly different between the four groups ( $p=0.039$, Kruskal-Wallis test) and analysis by the Cuzick test showed a highly significant $(p=0.005)$ decreasing trend in the ranked score of antiapoptotic activity across the four groups with increasing asthma severity and neutrophil counts (figure 2).

\section{Effect of mifepristone on neutrophilic apoptosis}

Mifepristone $(1 \mu \mathrm{M})$, an antagonist of the GC receptor, ${ }^{22}$ had no significant effect ( $p=0.608$ ) on constitutive neutrophil apoptosis alone $(n=6$, data not shown). In contrast, when used at $1 \mu \mathrm{M}$ it completely inhibited the apoptosis-delaying effect of dexamethasone (100 nM) in all experiments ( $\mathrm{n}=7$, data not shown). Mifepristone had no effect on the degree of neutrophil apoptosis when co-culturing sputum supernatants from SA, MA and HC in the absence or presence of mifepristone $(1 \mu \mathrm{M})$ (figure 3 ), excluding the possibility that the prosurvival effects of sputum from SA were mediated by residual GC present in sputum.

\section{Effects of inhibiting GM-CSF, TNF $\alpha$, LTB $_{4}$ and IL-8 on neutrophil survival in vitro}

In order to identify factors responsible for the prosurvival effect observed in the sputum from SA subjects, neutralising monoclonal antibodies (mAbs) against TNF $\alpha(100 \mu \mathrm{g} / \mathrm{ml})$ and GMCSF $(100 \mu \mathrm{g} / \mathrm{ml})$, as well as the selective antagonists for the IL-8 receptor, CXCR2 (SB-225002, $100 \mathrm{nM}$ ), and the $\mathrm{LTB}_{4}$ receptor, BLT1 (CP-105696, $10 \mu \mathrm{M}$ ), were applied in neutrophil cultures in the presence of sputum fluid phase. Neither the spontaneous

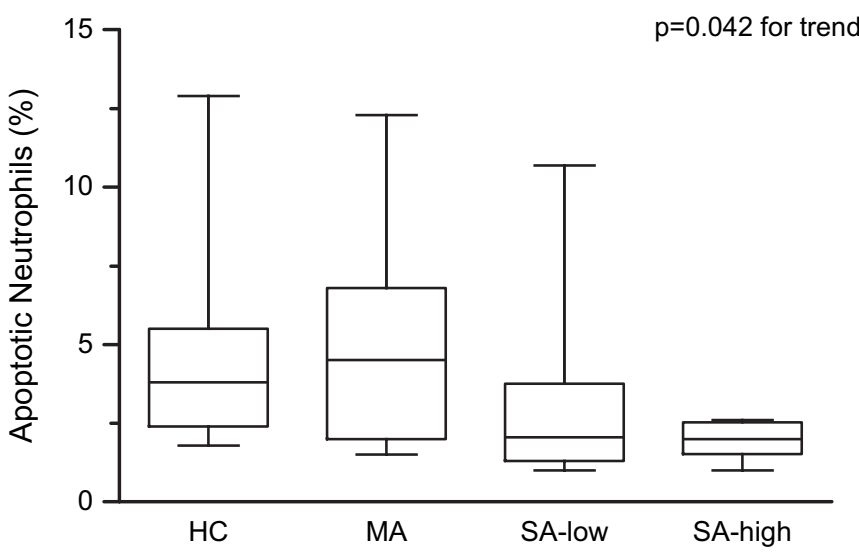

Figure 1 Proportions of apoptotic neutrophils in the sputum of subjects with severe asthma with high sputum neutrophil counts (SA-high), with severe asthma with low neutrophil counts (SA-low), with mild asthma (MA) and healthy controls (HC). Neutrophils were identified by immunohistochemical staining of sputum-derived cells with antineutrophil elastase antibody as described in the Methods section. Box and whisker plots show the numbers of apoptotic neutrophils expressed as percentages of total neutrophil counts. The boxes show the median and the 25th and 75th percentiles and the whiskers show the minimum to maximum values (Kruskal-Wallis test result $p=0.042$; Cuzick nonparametric test for trend $p=0.008$ ).

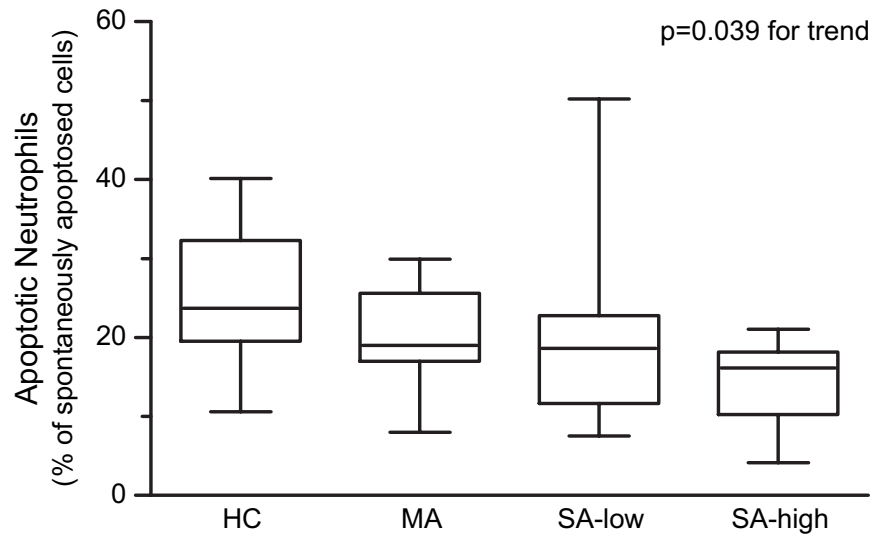

Figure 2 Antiapoptotic activity identified in sputum fluid phase of healthy controls $(\mathrm{HC})$, subjects with mild asthma (MA) and subjects with severe asthma with either low (SA-low) or high (SA-high) neutrophil counts. The extent of neutrophil apoptosis was assessed in cell culture of blood-derived neutrophils to which sputum fluid phase samples from the four subject categories were added as described in the Methods section. The results are shown as the numbers of apoptotic neutrophils in culture to which sputum fluid phase samples were added expressed as a percentage of spontaneous apoptosis (ie, numbers of apoptotic neutrophils counted in culture in RPMI medium alone). Box and whisker plots show the median, the 25th and 75th percentiles and the minimum to maximum values (Kruskal-Wallis test result $p=0.039$; Cuzick nonparametric test for trend $p=0.005$ ).

apoptosis in buffer control (figure 4A) nor the antiapoptotic effects of sputum (figure 4B) were significantly $(p=0.989$ and $\mathrm{p}=0.998$, respectively) inhibited by any of the selective receptor antagonists or neutralising $\mathrm{mAbs}$ in isolation or in combination.

\section{Study of the roles of signalling pathways and LPS in neutrophil survival mediated by sputum}

To elucidate the signalling pathways involved in the antiapoptotic effects mediated by sputum from SA subjects,

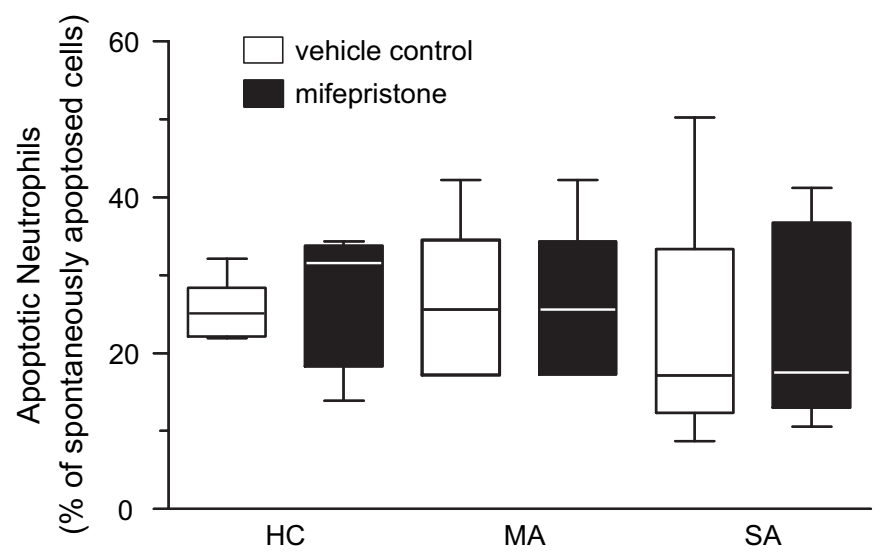

Figure 3 Effect of the glucocorticoid receptor antagonist, mifepristone $(1 \mu \mathrm{M})$, on neutrophil apoptosis in culture with sputum from subjects with severe asthma (SA) $(n=8)$, those with mild asthma (MA) $(n=5)$ and healthy controls $(\mathrm{HC})(n=6)$. Neutrophils were harvested after $22 \mathrm{~h}$ culture and apoptosis was assessed morphologically and expressed as a percentage of spontaneous apoptosis in medium alone incubated in the presence of mifepristone (solid bars) or vehicle control (open bars, ethanol $0.01 \%(\mathrm{v} / \mathrm{v})$ ) as indicated. Box and whisker plots show the median, the 25th and 75th percentiles and the minimum to maximum values. The effects of mifepristone were not significant in any of the subject groups. 




Figure 4 Neutralisation/blockade of sputum-mediated neutrophil survival. Application of the BLT1 antagonist, CP-105696 (10 $\mu \mathrm{M})$, or the CXCR2 antagonist, SB-225002 (100 nM), along with the antitumour necrosis $\alpha$ antibody (anti TNF $\alpha A b)(100 \mu \mathrm{g} / \mathrm{ml})$ or antigranulocyte-macrophage colony-stimulating factor (GM-CSF) $\mathrm{Ab}(100 \mu \mathrm{g} / \mathrm{ml})$, or with their isotype-matched immunoglobulin G (IgG) controls $(100 \mu \mathrm{g} /$ $\mathrm{ml})$, does not affect spontaneous, control apoptosis $(A)$ or sputum fluid phase-induced neutrophil survival (B). Data are shown as mean $\pm S D$ of four independent experiments, each performed in triplicate using peripheral blood neutrophils from different non-atopic healthy donors and sputum from subjects with severe asthma with high sputum neutrophil counts.

downstream events elicited by specific signalling pathways in neutrophils were investigated (figure 5). The antiapoptotic effects of sputum were not inhibited when using the long-acting phosphatidylinositol-3-kinase (PI3K) inhibitor, LY294002 $(30 \mu \mathrm{M})$. Similarly, polymyxin $\mathrm{B}(5 \mu \mathrm{g} / \mathrm{ml})$, which binds and inactivates LPS, had no effect on sputum-induced neutrophil survival, excluding a role for trace amounts of LPS in the sputum. Finally, since neutrophil chemotaxin receptors are coupled to $\mathrm{Gi} / \mathrm{o}$ proteins, we determined the effects of pertussis toxin (PTx; a Gi/o blocker) on sputum-mediated neutrophil survival. As shown in figure 5, pretreatment with PTx (100 ng/ $\mathrm{ml}, 2 \mathrm{~h}$ ) failed to abolish the inhibitory effects of sputum on neutrophil apoptosis in vitro.

\section{DISCUSSION}

This study has demonstrated the presence of prominent antiapoptotic activity for neutrophils in sputum samples in both healthy subjects and patients with asthma. Although a number of studies have investigated the characteristics of neutrophil apoptosis in induced sputum from subjects with asthma in vitro, this is the first investigation of the relationship between sputum neutrophil apoptosis and the prosurvival effect of the fluid phase of sputum on human neutrophils suggesting that prosurvival factors in the ELF are important for regulating the extent of airway neutrophilia in both health and disease. Of

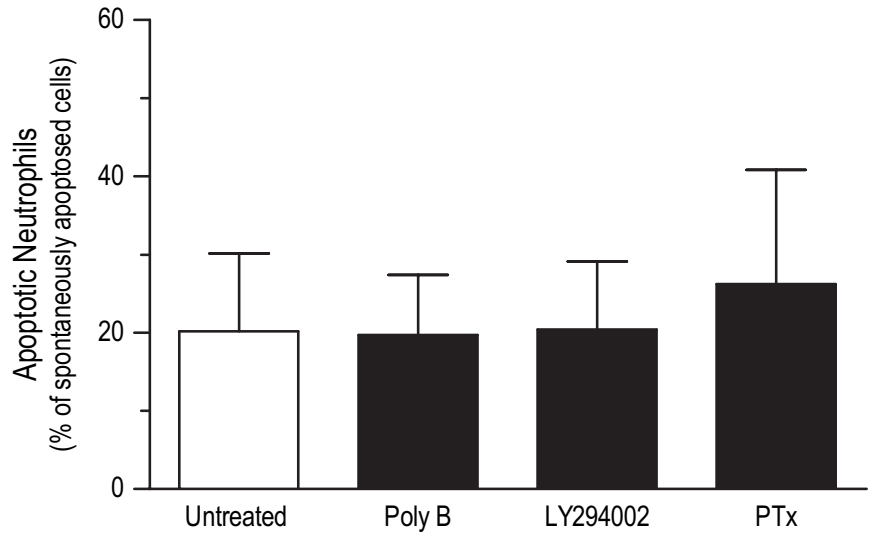

Figure 5 Sputum-induced neutrophil survival effect is not regulated by lipopolysaccharide (LPS), phosphatidylinositol-3-kinase (PI3K) or Gi/otype G-proteins. Neutrophils $\left(4 \times 10^{6} / \mathrm{ml}\right)$ were incubated in full RPMI culture medium alone, or with sputum from five subjects with severe asthma, applying sputum alone (Untreated) or sputum in the presence of the lipopolysaccharide (LPS)-neutralising agent, polymyxin B (Poly B; $5 \mu \mathrm{g} / \mathrm{ml})$, or using neutrophils pretreated with the PI3K inhibitor, LY294002 (30 $\mu \mathrm{M})$, or the Gi/o blocker, pertussis toxin (PTx; $100 \mathrm{ng} / \mathrm{ml}$, $2 \mathrm{~h}$ ). Data are the mean $\pm S D$ values of the percentages of apoptotic neutrophils.

relevance to the pathogenesis of severe asthma, the antiapoptotic activity was highest in patients with increased asthma severity that was associated with neutrophilia $(p=0.005$ for trend). However, extensive analysis of sputum factors that could be responsible for this phenomenon using selective inhibitors and blocking antibodies failed to attribute this activity to any of the proinflammatory cytokines, chemokines and lipid mediators that we studied.

Severe asthma has been a focus of investigation of many studies, but the mechanisms underlying its pathogenesis remain poorly understood. It is widely believed that severe asthma is not a single disease entity but consists of several pathologically distinct phenotypes. ${ }^{12}{ }^{13}$ Patients with severe asthma have persistent granulocytic inflammation and markedly differ in their airway cytokine/chemokine profile and their receptors when compared with those with mild or moderate asthma. ${ }^{4} 1415$ Significant advances have been made using sputum eosinophilia and neutrophilia as biomarkers of eosinophilic and neutrophilic forms of asthma, respectively, although the exact pathogenic role of these granulocytes remains unclear. Thus, eosinophilic asthma is usually viewed as being responsive to corticosteroids $^{29}$; indeed, studies have shown that corticosteroid treatment tailored on the basis of sputum eosinophil counts is superior to treatment guided by symptom control alone. ${ }^{30}$ Eosinophilia in patients with severe asthma is also associated with enhanced mast cell activation, T lymphocyte infiltration and production of profibrotic factors. ${ }^{12}$ Consistent with the latter, sputum eosinophilia has been associated with persistent airflow limitation, ${ }^{31}$ possibly because eosinophils are a major source of transforming growth factor $\beta$ (TGF $\beta$ ), a key profibrotic factor. ${ }^{32}$ In contrast, persistent neutrophilia is a feature of airway inflammation in patients with non-eosinophilic forms of asthma that are poorly responsive to corticosteroids. ${ }^{2} 1233$ The mechanisms underlying the accumulation of these two inflammatory cell phenotypes are unclear, although eosinophilia is largely explained by an overproduction of T helper (Th) type 2 cytokines that can be found in both atopic and non-atopic forms of asthma. ${ }^{34-36}$ It is widely recognised that neutrophilia is a consistent feature in asthma exacerbations in which context it 
could be a beneficial response to viral infection. In the current study, we excluded bacterial infection/colonisation (ie, all sputum samples were tested by routine bacterial culture methods). However, we did not look for evidence of persistent respiratory viral or Chlamydophila (formerly Chlamydia) infection, pathogens which have been associated with corticosteroid resistance (albeit in guinea-pigs) ${ }^{37}$ and greater decline in lung function in those with severe asthma. ${ }^{38}$

Neutrophils are key effector cells in innate immunity in the defence against bacterial, fungal and viral infections, so their increased presence in severe asthma does not necessarily imply that they cause harm. Inflammatory neutrophils can contribute to airway inflammation by virtue of their capacity to release cytotoxic proteases and reactive oxygen species. Such processes are considered to be a critical determinant in the development of acute respiratory distress syndrome ${ }^{39}$ and chronic obstructive pulmonary disease $e^{40}$ where they are associated with the decline in lung function and small airways dysfunction. ${ }^{41}$ It has been proposed that the neutrophilia seen in asthma is due to corticosteroid treatment as these agents directly promote neutrophil survival by inhibiting their apoptosis. ${ }^{18-20}$ In our study, we were unable to inhibit the prosurvival effect of sputum by using the GC receptor antagonist, mifepristone. While we cannot exclude an in vivo effect of corticosteroids, which could explain the reduced number of apoptotic neutrophils in severe neutrophilic asthma, this therapeutic in vivo effect cannot explain the observation of an ex vivo effect of factors present in the sputum fluid phase on blood-derived neutrophils.

Increases in concentrations of neutrophil-active factors, IL-8, $\mathrm{LTB}_{4}, \mathrm{GM}-\mathrm{CSF}$ and TNF $\alpha,{ }^{6-10}$ have been reported in both blood and airway secretions from subjects with asthma and we have recently shown these to be involved in promoting neutrophil chemotaxis mediated by the bronchial epithelium conditioned with epidermal growth factor. ${ }^{42}$ We postulated that these proinflammatory mediators might mediate the observed antiapoptotic activity of sputum, but this possibility was excluded by the ineffectiveness of specific neutralising/blocking agents for these neutrophil-active factors. We further investigated the intracellular signalling that might be involved in the observed survival-promoting activity and initially focused on PI3K which is known to modulate the survival responses of neutrophils. ${ }^{11}$ We found that the inhibition of apoptosis caused by the sputum fluid phase was not affected by the PI3K inhibitor, LY294002. We then sought to investigate whether the prosurvival activity is initiated by coupling of the receptor to a PTx-sensitive Gprotein, such as Gi or Go, and found that pretreatment with PTx $(100 \mathrm{ng} / \mathrm{ml})$ for $2 \mathrm{~h}$, optimally known to inhibit neutrophil functions mediated via these G-proteins, ${ }^{43}$ failed to block prosurvival effects induced by sputum. It was further speculated that the sputum might contain residual or contaminating levels of LPS which can also delay neutrophil apoptosis. ${ }^{20}$ However, we found no effects of the inactivator of endotoxins, polymyxin $\mathrm{B}$, arguing against the possibility that the prosurvival effects of sputum are mediated by contaminating LPS.

One possible explanation for the observed antiapoptotic effects of sputum is ATP released from dying airway cells. This premise is based on several lines of evidence that ATP has prosurvival activity for neutrophils and is released from dying cells, ${ }^{44} 45$ notably in chronic inflammatory conditions of the airways such as cystic fibrosis. ${ }^{46}$ Neutrophils themselves also release ATP, particularly under conditions of hypoxia, ${ }^{47}$ and ATP could act in an autocrine or paracrine manner to enhance neutrophil longevity at sites of inflammation. It would be interesting to determine, through a blocking approach, whether selective purinergic receptor antagonism is sufficient to prevent sputum-induced neutrophil survival. A further possible explanation for the observed antiapoptotic effects of sputum is that neutrophil survival may be prolonged because of respiratory viral infections and atypical bacterial pathogens such as Mycoplasma and Chlamydophila pneumoniae which are known to cause exacerbations of asthma. ${ }^{48-50}$ However, our study did not look at whether these respiratory pathogens are present in the sputum of those with severe asthma or whether interaction with these respiratory pathogens alters the bioactivity of sputum to modulate neutrophil survival programmes, a possibility that warrants further investigation. It is important to note, however, that none of the patients had had an exacerbation of asthma for at least 6 weeks.

One limitation of this study is the use of neutrophils from healthy donors, which raises the question of whether survival of neutrophils from patients with asthma might be different. Patients with asthma could not serve as donors of blood neutrophils since their blood often contains high eosinophil counts and there are no methods of neutrophil purification which can selectively deplete these cells. It would also have been desirable to study the interaction between the sputum fluid phase and neutrophils from sputum itself and from the same (autologous) donors, but this would have been technically difficult because of the large numbers of eosinophils that are also seen in neutrophil-enriched samples. This study should, therefore, be seen as a demonstration of increased proneutrophil survival activity in the ELF, leaving some uncertainty as to how exactly this heightened neutrophil apoptosis-resistant effect in the airways of those with severe asthma interacts with the neutrophils of the same patients.

In summary, this study has shown, for the first time, the presence of a prominent prosurvival activity in the airways of both healthy individuals and patients with asthma, the levels of which increase relative to the neutrophil counts and are highest in patients with severe neutrophilic asthma. Further investigation is required to fully elucidate the survival pathways regulated by these sputum-derived factors and how they contribute towards the apoptosis-resistant phenotype of neutrophils present in the airways of subjects with severe asthma.

Acknowledgements The authors wish to express their gratitude to the Flow Cytometry Unit and their staff, Dr Carolann McGuire and Dr Richard Jewell, for support with the flow cytometric analysis, the Southampton Histochemistry Research Unit for enabling the immunostaining, and the Southampton Wellcome Trust Clinical Research Facility for enabling the patient recruitment, assessment and sample collection.

Funding This research was funded by the Division of Infection, Inflammation and Immunity, University of Southampton School of Medicine.

Competing interests None.

Ethics approval The study was approved by the South and West Local Research Ethics Committee, Southampton, UK.

Provenance and peer review Not commissioned; externally peer reviewed.

\section{REFERENCES}

1. Wenzel SE, Szefler SJ, Leung DY, et al. Bronchoscopic evaluation of severe asthma. Persistent inflammation associated with high dose glucocorticoids. Am J Respir Crit Care Med 1997:156:737-43.

2. Green RH, Brightling CE, Woltmann G, et al. Analysis of induced sputum in adults with asthma: identification of subgroup with isolated sputum neutrophilia and poor response to inhaled corticosteroids. Thorax 2002;57:875-9.

3. Louis R, Djukanovic R. Is the neutrophil a worthy target in severe asthma and chronic obstructive pulmonary disease? Clin Exp Allergy 2006;36:563-7.

4. Louis R, Lau LC, Bron A0, et al. The relationship between airways inflammation and asthma severity. Am J Respir Crit Care Med 2000;161:9-16.

5. Teran LM, Campos MG, Begishvilli BT, et al. Identification of neutrophil chemotactic factors in bronchoalveolar lavage fluid of asthmatic patients. Clin Exp Allergy 1997;27:396-405. 
6. Jatakanon A, Uasuf C, Maziak W, et al. Neutrophilic inflammation in severe persistent asthma. Am J Respir Crit Care Med 1999;160:1532-9.

7. Vachier I, Bonnans $C$, Chavis $C$, et al. Severe asthma is associated with a loss of $\mathrm{LX}_{4}$, an endogenous anti-inflammatory compound. J Allergy Clin Immunol 2005;115:55-60.

8. Wenzel SE, Larsen $\mathrm{GL}$, Johnston $\mathrm{K}$, et al. Elevated levels of leukotriene $\mathrm{C}_{4}$ in bronchoalveolar lavage fluid from atopic asthmatics after endobronchial allergen challenge. Am Rev Respir Dis 1990;142:112-19.

9. Brown PH, Crompton GK, Greening AP. Proinflammatory cytokines in acute asthma. Lancet 1991;338:590-3.

10. Howarth PH, Babu KS, Arshad HS, et al. Tumour necrosis factor (TNF-alpha) as a novel therapeutic target in symptomatic corticosteroid dependent asthma. Thorax 2005;60:1012-18.

11. Murray J, Barbara JA, Dunkley SA, et al. Regulation of neutrophil apoptosis by tumor necrosis factor-alpha: requirement for TNFR55 and TNFR75 for induction of apoptosis in vitro. Blood 1997;90:2772-83.

12. Wenzel SE, Schwartz LB, Langmack EL, et al. Evidence that severe asthma can be divided pathologically into two inflammatory subtypes with distinct physiologic and clinical characteristics. Am J Respir Crit Care Med 1999;160:1001-8.

13. ENFUMOSA. Cross-sectional European multicentre study of the clinical phenotype of chronic severe asthma. European network for understanding mechanisms of severe asthma. Eur Respir J 2003;22:470-7.

14. Oiu Y, Zhu J, Bandi V, et al. Bronchial mucosal inflammation and upregulation of CXC chemoattractants and receptors in severe exacerbations of asthma. Thorax 2007:62:475-82.

15. Shannon J, Ernst $P$, Yamauchi $Y$, et al. Differences in airway cytokine profile in severe asthma compared to moderate asthma. Chest 2008;133:420-6.

16. Puddicombe SM, Polosa R, Richter A, et al. Involvement of the epidermal growth factor receptor in epithelial repair in asthma. FASEB J 2000;14:1362-74.

17. Hamilton LM, Puddicombe SM, Dearman RJ, et al. Altered protein tyrosine phosphorylation in asthmatic bronchial epithelium. Eur Respir J 2005;25:978-85.

18. Nguyen LT, Lim S, Oates T, et al. Increase in airway neutrophils after oral but not inhaled corticosteroid therapy in mild asthma. Respir Med 2005;99:200-7.

19. Zhang X, Moilanen E, Kankaanranta H. Beclomethasone, budesonide and fluticasone propionate inhibit human neutrophil apoptosis. Eur J Pharmacol 2001;:431:365-71.

20. Meagher LC, Cousin JM, Seckl JR, et al. Opposing effects of glucocorticoids on the rate of apoptosis in neutrophilic and eosinophilic granulocytes. J Immunol 1996:156:4422-8.

21. Haslett C. Granulocyte apoptosis and inflammatory disease. Br Med Bull 1997; 53:669-83

22. Agarwai MK. The antiglucocorticoid action of mifepristone. Pharmacol Ther 1996; 70:183-213.

23. Belda J, Leigh R, Parameswaran K, et al. Induced sputum cell counts in healthy adults. Am J Respir Crit Care Med 2000;161:475-8.

24. Paggiaro PL, Chanez P, Holz 0, et al. Sputum induction. Eur Respir J 2002; $37: 3 s-8 s$

25. Yoshikawa T, Dent G, Ward J, et al. Impaired neutrophil chemotaxis in chronic obstructive pulmonary disease. Am J Respir Crit Care Med 2007;175:473-9.

26. Pulford KA, Erber WN, Crick JA, et al. Use of monoclonal antibody against human neutrophil elastase in normal and leukaemic myeloid cells. J Clin Pathol 1988:41:853-60.

27. Rytila P, Plataki M, Bucchieri F, et al. Airway neutrophilia in COPD is not associated with increased neutrophil survival. Eur Respir J 2006;28:1163-9.

28. Cuzick J. A Wilcoxon-type test for trend. Stat Med 1985:4:87-90.
29. Barnes PJ. The role of inflammation and anti-inflammatory medication in asthma. Respir Med 2002;96(Suppl A):S9-15

30. Green RH, Brightling CE, McKenna $S$, et al. Asthma exacerbations and sputum eosinophil counts: a randomised controlled trial. Lancet 2002;360:1715-21.

31. ten Brinke A, Zwinderman AH, Sterk PJ, et al. Factors associated with persisten airflow limitation in severe asthma. Am J Respir Crit Care Med 2001:164:744-8.

32. Minshall EM, Leung DY, Martin RJ, et al. Eosinophil-associated TGF-beta1 mRNA expression and airways fibrosis in bronchial asthma. Am J Respir Cell Mol Biol 1997:17:326-33

33. in't Veen JC, Smits HH, Hiemstra PS, et al. Lung function and sputum characteristics of patients with severe asthma during an induced exacerbation by double-blind steroid withdrawal. Am J Respir Crit Care Med 1999:160:93-9.

34. Bousquet J, Chanez $P$, Lacoste JY, et al. Eosinophilic inflammation in asthma. N Engl J Med 1990;323:1033-9.

35. Robinson DS, Hamid Q, Ying S, et al. Predominant Th2-like bronchoalveolar T-lymphocyte population in atopic asthma. N Engl J Med 1992;326:298-304.

36. Humbert M, Ying S, Corrigan C, et al. Bronchial mucosal expression of the genes encoding chemokines RANTES and MCP-3 in symptomatic atopic and nonatopic asthmatics: relationship to the eosinophil-active cytokines interleukin (IL)-5, granulocyte macrophage-colony-stimulating factor, and IL-3. Am J Respir Cell Mol Biol 1997; 16:1-8

37. Yamada K, Elliott WM, Brattsand R, et al. Molecular mechanisms of decreased steroid responsiveness induced by latent adenoviral infection in allergic lung inflammation. J Allergy Clin Immunol 2002;109:35-42.

38. Black PN, Scicchitano R, Jenkins CR, et al. Serological evidence of infection with Chlamydia pneumoniae is related to the severity of asthma. Eur Respir $\mathrm{J}$ 2000; 15:254-9.

39. MacNee W. Pulmonary neutrophil kinetics. Clin Phys Physiol Meas 1990;11(Suppl A):133-9.

40. Barnes PJ. Mechanisms in COPD: differences from asthma. Chest 2000:117:10S-14S

41. O'Donnell RA, Peebles C, Ward JA, et al. Relationship between peripheral airway dysfunction, airway obstruction, and neutrophilic inflammation in COPD. Thorax 2004;59:837-42.

42. Uddin M, Seumois G, Lau LC, et al. Enhancement of neutrophil function by the bronchial epithelium stimulated by epidermal growth factor. Eur Respir J 2008;31:714-24.

43. Spangrude GJ, Sacchi F, Hill HR, et al. Inhibition of lymphocyte and neutrophil chemotaxis by pertussis toxin. J Immunol 1985;135:4135-43.

44. Dubyak GR, el-Moatassim C. Signal transduction via P2-purinergic receptors for extracellular ATP and other nucleotides. Am J Physiol 1993;265:C577-606.

45. Vaughan KR, Stokes L, Prince LR, et al. Inhibition of neutrophil apoptosis by ATP is mediated by the P2Y11 receptor. J Immunol 2007;179:8544-53.

46. Donaldson SH, Lazarowski ER, Picher M, et al. Basal nucleotide levels, release, and metabolism in normal and cystic fibrosis airways. Mol Med 2000;6:969-82

47. Eltzschig HK, Abdulla P, Hoffman E, et al. Hif-1-dependent repression of equilibrative nucleoside transporter (ENT) in hypoxia. J Exp Med 2005:202:1493-505.

48. Johnston SL, Pattemore PK, Sanderson G, et al. Community study of role of viral infections in exacerbations of asthma in 9-11 year old children. Br Med $\mathrm{J}$ 1995:310:1225-9.

49. Corne JM, Marshall C, Smith S, et al. Frequency, severity, and duration of rhinovirus infections in asthmatic and non-asthmatic individuals: a longitudinal cohort study. Lancet 2002;359:831-4.

50. Sutherland ER, Martin RJ. Asthma and atypical bacterial infection. Chest 2007:132:1962-6. 